\title{
Modeling of the Doping Dependent Superconducting Transition Temperature for Cuprates: Role of Antiferromagnetic Fluctuations
}

\author{
S. H. Naqib ${ }^{1}$ \\ Department of Physics, Rajshahi University, Rajshahi-6205, Bangladesh \\ Received 17 September 2008, in final form 24 October 2008
}

\begin{abstract}
We have calculated the superconducting transition temperature, $T_{c}$, of high- $T_{c}$ cuprates within the strong-coupling Bardeen-Cooper-Schrieffer (BCS) formalism. The hole pairing was attributed to the antiferromagnetic spin fluctuations. Such fluctuations are believed to exist over the whole superconducting hole concentrations, $p$, extending from the underdoped to the overdoped regimes. A complete consideration of the $p$-dependences of the antiferromagnetic spin excitation spectral weight, spin fluctuation frequency, $\omega_{s f}$, and the effect of the pseudogap on the electronic density of states seems to reproduce the experimental $T_{c}(p)$ satisfactorily over the entire doping range. The possible implications of this finding are discussed in detail.
\end{abstract}

Keywords: Cuprate superconductors; Superconducting $T_{c}$; Spin fluctuations; Pseudogap.

(C) 2009 JSR Publications. ISSN: 2070-0237 (Print); 2070-0245 (Online). All rights reserved.

DOI: 10.3329 /jsr.vlil.1181

\section{Introduction}

Elucidation of the physical mechanism leading to cuprate superconductivity remains as one of the most outstanding issues in the field of condensed matter physics, still after more than two decades of its discovery [1]. The non Fermi-liquid (FL) like behavior of the normal state properties [2-5] are posing too hard a hurdle for the theoreticians to develop a proper formalism describing the underlying physics of these strongly correlated electronic systems, so far. The normal and superconducting (SC) state properties, including $T_{c}$, of cuprates belonging to different families are most sensitive to the number of doped hole carriers per $\mathrm{CuO}_{2}$ plane, $p[4,6]$. Besides the high value of $T_{c}$ at the optimum hole content, two of the most robust $p$-dependent features observed in all the cuprate superconductors are the presence of antiferromagnetic (AF) correlations and of the pseudogap (PG) in the low-energy quasiparticle (QP) excitation spectrum. The undoped parent compounds can be described as Mott-Hubbard insulators with long range AF ordering. Introducing holes in the $\mathrm{CuO}_{2}$ planes quickly destroys this long range magnetic ordering and for most of the

${ }^{1}$ Email: salehnaqib@yahoo.com 
cuprates a spin-glass state appears before the ground state become SC with further hole doping. Short range (SR) AF correlation coexists with superconductivity [7-9], but this SR correlation becomes progressively weaker as $p$ is increased $[9,10]$. One of the most extensively investigated phenomena in the physics of cuprate superconductors is the socalled pseudogap [10-12]. It is widely believed that an understanding of the PG will lead to the understanding of the mechanism of superconductivity itself. The pseudogap correlation is observed in the $T$ - $p$ phase diagram over a certain doping range, extending from the UD to the slightly OD regions. In the PG state a number of anomalies are observed both in normal and superconducting states where contrary to one of the central tenants of the Landau Fermi-liquid theory, low-energy QP excitations are gapped along certain directions of the Brillouin zone while Fermi-arcs exist in other directions [12, 13]. As a result the average low-energy QP spectral density is significantly depleted in the UD compounds. Considerable debate has ensued as to the nature of the PG and the experimental and theoretical situations are thought to be rather inconclusive at this moment [10-12].

Ever since the discovery of high- $T_{c}$ superconductivity in cuprates, a large number of theoretical studies [14-19] have been carried out to describe the parabolic $p$-dependence of $T_{c}$ [20], with mixed degrees of success. In this paper we have adopted a semi-empirical approach. We have developed a minimal model to calculate $T_{c}$ based on the experimental doping dependences of two of the universal features associated with cuprates, the SR AF fluctuations and the PG, as mentioned above. Here we have assumed that AF spin fluctuations are responsible for Cooper pairing, the energy cut-off is determined from the combined effects of doping dependent spin fluctuation energy and the spectral weight associated with the spin excitation spectrum. The magnitude of the $p$-dependent PG energy scale plays a crucial role as it determines the electronic density of states (EDOS) near the Fermi-level, and consequently the electron-boson (magnon modes in the present study) coupling constant, $\lambda$. The strong-coupling BCS formalism reproduces a nearly parabolic $T_{c}(p)$ with values close to the experimental ones, when the observed $p$ dependences of spin and PG correlations are properly considered. The possible implications of our finding are discussed in detail in section 3.

\section{Formalism and Results}

The expression for the SC transition temperature was obtained following earlier works $[21,22]$ using the thermal Green's function and the generalized Eliashberg equation. The hole pairing was mediated by the bosonic excitations describing the collective spin fluctuations. For simplicity we have considered only the $s$-wave order parameter, even though the high- $T_{c}$ cuprates show predominantly $d$-wave characteristics. As far as $T_{c}$ is concerned, this only has a moderate effect. For example the gap ratio, $2 \Delta(0) / k_{B} T_{c}=3.53$, for canonical $s$-wave superconductors, whereas it is 4.28 for canonical $d$-wave superconductors [23]. Here $\Delta(0)$ is the zero- $T$ uniform SC gap and the maximum SC gap in the $s$-wave and $d$-wave scenarios, respectively. The $T_{c}$ equation is given below $[21,22]$. 


$$
T_{c}=\frac{2 \exp (\delta)}{\pi} \omega_{c} \exp \left[-\frac{1+\lambda}{\lambda-\mu}\right]
$$

where $\delta$ is the Euler's constant, $\omega_{c}$ is the cut-off energy, $\lambda$ denotes the electron-boson coupling constant, and $\mu$ is the attractive coulombic pseudopotential. In general $\mu<<1$ [21] and can be neglected in the strong coupling regime. It is important to note that, unlike in the conventional phonon-mediated superconductors, for cuprates $\omega_{c}$ should not be taken as the characteristic boson energy, $\omega_{s f}$. This is because of the strong doping dependence of the spin spectral weight. For example, the imaginary part of the dynamical spin susceptibility, $\operatorname{Im} \chi(q, \omega)$, probed by inelastic neutron scattering (INS), decreases systematically with increasing hole content [24]. Therefore, as a first approximation, $\omega_{c}$ and $\omega_{s f}$ can be related as $\omega_{c}=\eta \omega_{s f}$, where $\eta$ is a $p$-dependent constant taking into account the proper spin fluctuation spectral weight at a particular doping. Generally, $\omega_{s f} \sim 1 / \xi_{A F}{ }^{2}$ [19], where $\xi_{A F}$ is the AF correlation length, and for cuprate superconductors, the experimental $q$-widths of the INS peaks at the AF wave vector show an almost linear dependence on $T_{c}$ up to the optimum doping [24]. This indicates that $\xi_{A F}$ decreases monotonically with increasing $p$ and therefore $\omega_{s f}$ increases as hole content increases. On the other hand $\eta$ obtained from energy integrated $\operatorname{Im} \chi(q, \omega)$ showed a much stronger $p$ dependence [24]. Additional complexity arises from the fact that $\xi_{A F}$ and consequently $\omega_{s f}$ is $T$-dependent. To calculate $T_{c}$, we have used $\omega_{s f}\left(T_{c}\right)$ for determining $\omega_{c}$. Considering all these and using the available experimental data $[2,9,19,24,25]$ the variation of $\omega_{c}$ with hole content for $\mathrm{YBa}_{2} \mathrm{Cu}_{3} \mathrm{O}_{7-\delta}(\mathrm{Y} 123)$, the most widely studied cuprate superconductor, is shown in Fig. 1. The cut-off energy was found to decrease linearly with increasing $p$ and was set to zero at $p=0.27$, the value at which superconductivity vanishes in the OD side for most of the cuprate superconductors. Similar trends were found in earlier studies [17, 19]. The electron-boson coupling constant can be defined as $\lambda=N_{0} V$, where $N_{0}$ is the EDOS near the Fermi-level and $V$ is the effective pairing potential. For conventional BCS superconductors with Fermi-energy of the order of $10 \mathrm{eV}, N_{0}$ is almost constant over an energy width determined by the characteristic boson energy scale. For cuprates the situation is very different when PG is present. $N(\varepsilon)$ is severely depleted near the Fermilevel and the number of holes participating in pairing is significantly reduced. As a consequence the value of $\lambda$ is also reduced even if the pairing potential is stronger in the UD side (since the short range Coulomb repulsion/AF exchange energy increases with decreasing $\mathrm{p}$, leading to stronger electronic correlations). In the presence of the PG, the EDOS can be modeled as [26, 27]

$$
\begin{array}{rlrlrl}
N(\varepsilon) & =N_{0}\left|\varepsilon / \varepsilon_{g}\right| & & \text { for } & & \varepsilon \leq \varepsilon_{g} \\
& =N_{0} & \text { for } & \varepsilon>\varepsilon_{g}
\end{array}
$$

Here $\varepsilon_{g}$ is the PG energy scale. Such a triangular PG was also used successfully by Loram et al. [28] to describe their pioneering electronic heat capacity results. Using the experimental values of the $\varepsilon_{g}(p)[6,29]$ and a gradually decreasing effective pairing potential with increasing hole content, we have calculated the values of $\lambda$ as a function of 
$p$. This variation of $\lambda$ with hole concentration is illustrated in Fig. 2. It is not surprising that $\lambda(p)$ resembles qualitatively to the $p$-dependent superfluid density of cuprates [10], as the magnitude of the PG primarily determines both these parameters. It is also worth mentioning that recent angle-resolved photoemission spectroscopy (ARPES) indicates that strong coupling features are stronger in the UD compounds [30, 31], whereas Fig. 2 shows that $\lambda$ decreases in the UD side. The reason for this apparent contradiction is discussed in the next section. For the optimally and overdoped compounds the values of $\lambda$ agree reasonably well with other studies [30-32].

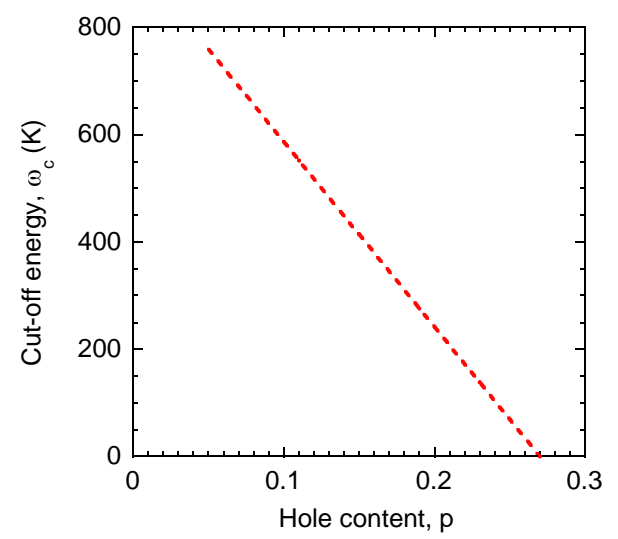

Fig. 1. Variation of the characteristic cut-off energy (expressed in the units of temperature) with the hole concentration.

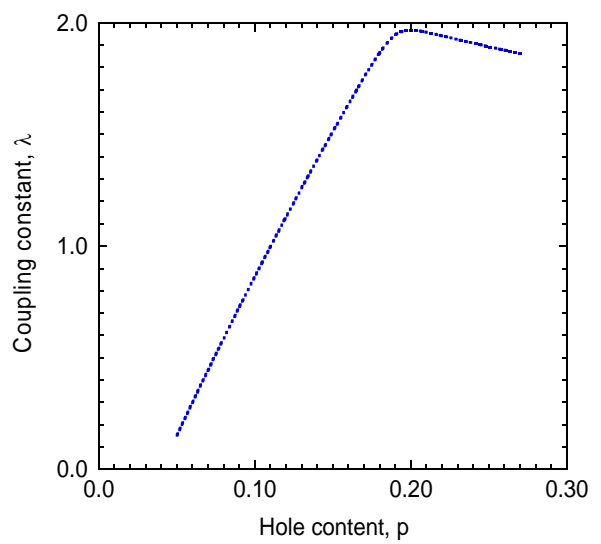

Fig. 2. Variation of the electron-boson coupling constant with hole concentration (see text for details).

The variation of the superconducting transition temperature with $p$ obtained from Eq. (1), using the $p$-dependent values of $\omega_{c}$ and $\lambda$, is shown in Fig. 3. In the inset of the same figure we have shown the experimental parabolic $T_{c}(p)$ (for Y123, Bi2212 and other $90 \mathrm{~K}$ 
superconductors). Considering the simplicity of the model used in this study, the agreement is quite remarkable.

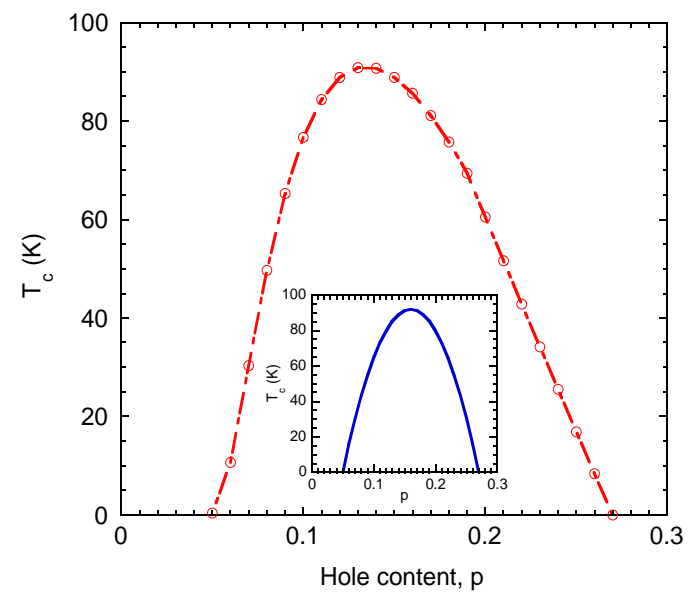

Fig. 3. Main panel: Calculated $T_{c}$ versus $p$. Inset: The full blue line was obtained using the parabolic $T_{c}(p)$ equation [20] with a maximum $T_{c}=92 \mathrm{~K}$ at $p=0.16$ (as found experimentally for Y123 and Bi2212).

\section{Discussion and Conclusions}

The central result of the current study is shown in Fig. 3. Despite the impressive agreement between the theoretically calculated $T_{c}$ and their experimental values, a number of features associated with the formalism used need elaborate discussion. None of the previous theoretical studies on the $p$-dependence of $T_{c}$ [14-19] took into account of the possible role of PG on the carrier-boson coupling constant. High values of $\lambda$ can be extracted from the kinks in the momentum distribution curves (MDC) of ARPES measurements [33] for the UD cuprates. The important point here is the fact that this kink is seen at an energy $\sim 70 \mathrm{meV}(\sim 800 \mathrm{~K}$, if expressed in the temperature scale $)$. This energy is almost certainly larger than the PG energy scale and the large coupling constant arises from the complete recovery of the outside-PG EDOS (see Eq. (2)). For example, in this paper we have taken $\lambda=0.16$ for the very UD composition $(p=0.05)$. If the fully developed EDOS outside the PG is considered then this becomes 2.27, larger than the highest value shown in Fig. 2. It is true that the spectral weight associated with the spin excitations are largely reduced in the OD side and this has been taken against any spinfluctuation model for superconductivity in cuprates [4]. Here, in favor of the spinfluctuation model, it can be said that even if the spectral weight is greatly reduced, the phase stiffness and therefore the strength of phase coherence is quite large in the OD side (compared to the UD region) and this is one of the deciding factors for superconductivity to occur [34]. 
We have ruled out phonons as the collective mode mediating pairing from the outset. Interpretations of the kinks seen in ARPES experiments and the observed isotope effects in the MDC [35] have generated considerable interest regarding the possible role played by the phonons [36]. We still think that a $d$-wave pairing is unlikely in phonon mediated superconductors. A magnetic pairing on the other hand can lead to line nodes in the SC energy gap quite easily due to Fermi-surface nesting as found in cuprates [37]. The observed isotope effect can be related to the so-called spin-charge stripe correlations [38]. When such spin and charge orderings are present, lattice and charge degrees of freedoms influence each other quite strongly [39] and any isotopic substitution should affect the QP energy dispersion.

It is seen from Fig. 3 that the calculated $T_{c}(p)$ is slightly asymmetric. $T_{c}(p)$ rises at a faster rate in the UD side. Also the maximum of calculated $T_{c}(p)$ is attained at $p \sim 0.145$, whereas for most of the pure cuprate superconductors the value of $p$ for maximum $T_{c}$ is 0.16 . These discrepancies can be largely removed by taking into account of the proper values of coulombic pseudopotential and the existence of the van-Hove singularity (vHS) in the EDOS [40]. We have taken $\mu=0$ in this study, because a reliable estimate of this parameter for cuprates as a function of doping is still not available. Nevertheless it is expected that $\mu$ should increase as $p$ decreases as a result of poor charge screening in the UD side. This should reduce $T_{c}$ in the UD region and can make the calculated $T_{c}(p)$ more symmetric. The presence of an extended van-Hove saddle point singularity [40] on the other hand can shift the optimum doping to higher values. In this scenario the Fermi-level approaches the peak in the EDOS due to the vHS as hole content increases and the Fermienergy, $\varepsilon_{F}$, crosses this peak in the OD side [41]. If such an additional enhancement of the EDOS is considered as $p$ increases, the maximum of the $T_{c}(p)$ would occur at $p>0.145$ within the present formalism. It should be mentioned that the existence of the vHS and the shift of the Fermi-level with doping have been invoked to describe the $T_{c}(p)$ behavior in cuprates before [14]. In such a picture $\varepsilon_{F}$ always crosses the vHS at the optimum doping. This is in contradiction with experiments, where the crossing occurs in the deeply OD region at $p \sim 0.225[41]$.

In summary, we have used a minimal model to describe the doping dependence of $T_{c}$ for cuprate superconductors. A simple strong-coupling BCS formalism with an $s$-wave order parameter reproduces a nearly parabolic $T_{c}(p)$ when the doping dependent values of the cut-off energy and the PG are used. The qualitative and quantitative agreements are quite good considering the simplicity of the approach. This analysis is far from complete. It would be interesting to study the effects of $d$-wave pairing, coulombic pseudopotential, and the vHS in the EDOS within the spin fluctuation model in a future study. Finally, the strong pairing energy and high values of $\lambda$ can lead to the formation of spin-polarons and it would be interesting to investigate the possible implications.

\section{Acknowledgements}

The author acknowledges Prof. J. R. Cooper, Dr. J. W. Loram from University of Cambridge, UK, and Prof. J. L. Tallon, Victoria University, Wellington, New Zealand, for useful discussions at various stages. 


\section{References}

1. J. G. Bednorz and K. A. Müller, Z. Phys B 64, 189 (1986). doi:10.1007/BF01303701

2. P. Monthoux and D. Pines, Phys. Rev. B 50, 16015 (1994). doi:10.1103/PhysRevB.50.16015

3. C. M. Varma, Phys. Rev. Lett. 83, 3538 (1999). doi:10.1103/PhysRevLett.83.3538

4. P. A. Lee, Rep. Prog. Phys. 71, 012501 (2008) and references therein. doi:10.1088/0034-4885/71/1/012501

5. P. W. Anderson, Science 235, 1196 (1987). doi:10.1126/science.235.4793.1196

6. S. H. Naqib, J. R. Cooper, J. L. Tallon, and C. Panagopoulos, Physica C 387, 365 (2003). doi:10.1016/S0921-4534(02)02330-4

7. C. Panagopoulos, and V. Dobrosavljević, Phys. Rev. B 72, 014536 (2005). doi:10.1103/PhysRevB.72.014536

8. S. Wakimoto, H. Zhang, K. Yamada, I. Swainson, Hyunkyung Kim, and R. J. Birgeneau, Phys. Rev. Lett. 92, 217004 (2004).doi:10.1103/PhysRevLett.92.217004

9. S. Pailhés, C. Ulrich, B. Fauqué, V. Hinkov, Y. Sidis, A. Ivanov, C. T. Lin, B. Keimer, and P. Bourges, Phys. Rev. Lett. 96, 257001 (2006). doi:10.1103/PhysRevLett.96.257001

10. J. L. Tallon and J. W. Loram, Physica C 349, 53 (2001). doi:10.1016/S0921-4534(00)01524-0

11. V. Z. Kresin, Y. N. Ovchinnikov, and S. A. Wolf, Phys. Rep. 431, 231 (2006). doi:10.1016/j.physrep.2006.05.006

12. M. R. Norman, D. Pines, and C. Kallin, Adv. Phys. 54, 715 (2005). doi:10.1080/00018730500459906

13. A. Damascelli, Z. Hussain, and Z. -X. Shen, Rev. Mod. Phys. 75, 473 (2003). doi:10.1103/RevModPhys.75.473

14. D. M. Newns, H. R. Krishnamurthy, P. C. Pattnaik, C. C. Tsuei, and C. L. Kane, Phys. Rev. Lett. 69, 1264 (1992). doi:10.1103/PhysRevLett.69.1264

15. L. Pietronero, S. Strässler, and C. Grimaldi, Phys. Rev. B 52, 10516 (1995); C. Grimaldi, L. Pietronero, and S. Strässler, Phys. Rev. B 52, 10530 (1995).

16. M. Langer, J. Schamalian, S. Grabowski, and K. H. Bennemann, Phys. Rev. Lett. 75, 4508 (1995). doi:10.1103/PhysRevLett.75.4508

17. P. Monthoux and D. Pines, Phys. Rev. B 47, 6069 (1993). doi:10.1103/PhysRevB.47.6069

18. X. J. Chen and H. Q. Lin, Phys. Rev. B 69, 104518 (2004). doi:10.1103/PhysRevB.69.104518

19. P. Monthoux and D. Pines, Phys. Rev. B 49, 4261 (1994). doi:10.1103/PhysRevB.49.4261

20. M. R. Presland, J. L. Tallon, R. G. Buckley, R. S. Liu, N. E. Fowler, Physica C 176, 95 (1991). doi:10.1016/0921-4534(91)90700-9

21. J. P. Carbotte, Rev. Mod. Phys. 62, 1027 (1990).doi:10.1103/RevModPhys.62.1027

22. A. K. M. A. Islam and S. H. Naqib, J. Phys. Chem. Solids 58, 1153 (1997). doi:10.1016/S0022-3697(96)00145-X

23. Tifrea, I. Grosu, and M. Crisan, Physica C 371, 104 (2002). doi:10.1016/S0921-4534(01)01072-3

24. P. Bourges in Neutron Scattering in Novel Materials, Ed. A. Furrer, World Scientific, 2000.

25. V. Chubukov, D. Pines, and J. Schamalian in The Physics of Conventional and Unconventional Superconductors, Eds. K. H. Bennemann and J. B. Ketterson, Springer, New York, 2002.

26. S. H. Naqib and R. S. Islam, Supercond. Sci. Tech. 21, 105017 (2008). doi:10.1088/0953-2048/21/10/105017

27. S. H. Naqib and J. R. Cooper, Physica C 460-462, 750 (2007).doi:10.1016/j.physc.2007.03.168

28. J. W. Loram, J. Luo, J. R. Cooper, W. Y. Liang, and J. L. Tallon, J. Phys. Chem. Solids 62, 59 (2001). doi:10.1016/S0022-3697(00)00101-3

29. S. H. Naqib, J. R. Cooper, J. L. Tallon, R. S. Islam, and R. A. Chakalov, Phys. Rev. B 71, 054502 (2005). doi:10.1103/PhysRevB.71.054502

30. A. S. Alexandrov, Theory of Superconductivity: From Weak to Strong Coupling, IoP and Taylor and Francis Publishers, Bristol, 2003. 
31. A. A. Kordyuk, S. V. Borisenko, V. B. Zabolotnyy, J. Geck, M. Knupfer, J. Fink, B. Büchner, C. T. Lin, B. Keimer, H. Berger, A. V. Pan, S. Komiya, and Y. Ando, Phys. Rev. Lett. 97, 017002 (2006). doi:10.1103/PhysRevLett.97.017002

32. J. C. Campuzano, M. R. Norman, and M. Randeria, condmat/0209476v1 and the references therein.

33. A. Lanzara, P. V. Bogdanov, X. J. Zhou, S. A. Keller, D. L. Feng, E. D. Lu, T. Yoshida, H. Eisaki, A. Fujimori, K. Kishio, J. -I. Shimoyama, T. Noda, S. Uchida, Z. Hussain, and Z. -X. Shen, Nature 412, 510 (2001). doi:10.1038/35087518

34. V. J. Emery and S. A. Kivelson, Nature 374, 4347 (1995). doi:10.1038/374434a0

35. G. -H. Gweon, T. Sasagawa, S. Y. Zhou, J. Graf, H. Takagi, D. -H. Lee, and A. Lanzara, Nature 430, 187 (2004). doi:10.1038/nature02731

36. S. Ishihara and N. Nagaosa, Phys. Rev. B 69, 144520 (2004). doi:10.1103/PhysRevB.69.144520

37. D. Pines, Z. Phys. B 103, 129 (1997).

38. J. M. Tranquada, B. J. Sternlib, J. D. Axe, Y. Nakamura, and S. Uchida, Nature 375, 561 (1995). doi:10.1038/375561a0

39. E. W. Carlson, V. J. Emery, S. A. Kivelson, and D. Orgad in The Physics of Superconductors Vol. II. Superconductivity in Nanostructure, High-T $T_{c}$ and Novel Superconductors, Organic Superconductors, Eds. K. H. Bennemann, J. B. Ketterson, Springer 2004.

40. J. Ma, C. Quitmann, R. J. Kelly, P. Almeras, H. Berger, G. Margaritondo, and M. Onellion, Phys. Rev. B 51, 3832 (1995). doi:10.1103/PhysRevB.51.3832

41. A. Kaminski, S. Rosenkranz, H. M. Fretwell, M. R. Norman, M. Randeria, J. C. Campuzano, J. M. Park, Z. Z. Li, and H. Raffy, Phys. Rev. B 73, 174511 (2006).

doi:10.1103/PhysRevB.73.174511 